\title{
A valid and reliable tool to assess nursing students`clinical performance
}

\author{
Sharareh Khosravi*, Mehrnoosh Pazargadi, Tahereh Ashktorab, Hamid Alavi majd \\ *PhD of Nursing, School of Nursing \& Midwifery, Arak University of Medical Sciences, Tehran, Iran \\ PhD of Educational Management, School of Nursing \& Midwifery, Shahid Beheshti University of Medical Sciences, Tehran, Iran \\ PhD of Nursing, School of Nursing \& Midwifery, Shahid Beheshti University of Medical Sciences, Tehran, Iran \\ PhD of Biostatistics, Department of Biostatistics, School of Paramedical Sciences, Shahid Beheshti University of Medical Sciences, \\ Tehran, Iran \\ *Corresponding authorE-mail: khosravi@arakmu.ac.ir
}

\begin{abstract}
Purpose: This study was conducted to design a valid and reliable assessment tool for evaluating nursing students` performance in clinical education.

Approaches: In this methodological study considering nursing students` performance definition; the tool, its domains and items were specified through expert interviews and literature review. To determine validity; face validity (using expert viewpoints and item impact method) and content validity (using expert viewpoints, content validity ratio and content validity index) were considered. Reliability was evaluated through Cronbach`s alpha and interclass correlation (ICC).

Results: Seven domains and 55 items were found and the primary tool was designed. Item impact score and content validity ratio were 1.5 and more and 0.54 and more for each item, respectively and total content validity index was 0.94 . Cronbach`s alpha for the whole tool was 0.992 and ICC was 0.89 .

Conclusions: The designed tool with a high validity and reliability is considered proper and can be used for evaluating nursing students`clinical performance.
\end{abstract}

Keywords: assessment tool, clinical education, clinical evaluation, nursing student

\section{Introduction}

Nursing education involves both theoretical and practical training processes $[1,2]$ and as a practice-based discipline, learning must be mostly in clinical settings with qualified educators [3, 4]. Clinical education is an essential and indispensable part of professional nursing education [2,3]. It provides nursing students with opportunities to acquire professional knowledge, skills, and competencies for patient care and thinking and acting like professionals [5, 6].

Evaluation is central to any educational program, but is particularly critical for nursing education to ensure that nurses are safe and competent practitioners [7]. In clinical evaluation, educators assess the extent of the students' learning and quality of performance in clinical practice [8]. Clinical evaluation aims to facilitate the improvement of students into safe, ethical and accountable practitioners and to benchmark the students' progress towards achieving clinical learning objectives [1,9].

A recurrent theme in literature related to nursing students ' clinical evaluation is the need for assessment tools that are targeted, reliable and valid [10]. An efficient evaluation tool is essential in judging the clinical performance of nursing students. It is also important that the evaluation tool accurately assess the critical competencies which students must demonstrate in clinical settings. The tool should be unambiguous, succinct and adaptable to a wide variety of clinical experiences, and inform students and educators of students' progress [1, 11].

Despite the importance of valid, reliable and objective assessment tools, studies showed there are still many challenges in this area. It is said that instruments used to assess competence in nursing have not been rigorously tested reliability and validity so they have not the appropriate validity and reliability to evaluate students' performances [12, 13]. Also students have little confidence in their assessment tools. They believed that the tools are difficult to understand and most of the tools have little attention to necessary skills, leaving students with anxiety about their ability to perform important nursing skills [14]. 
This study was conducted to design a valid and reliable clinical performance evaluation tool for nursing students` clinical performance.

\section{Approaches}

This is a methodological research. Methodological research is the development and evaluation of data collection instruments, scales and techniques. It basically includes; defining the construct/concept or behavior to be measured, formulating the tool's items, and testing the tool's validity and reliability [15].

In this methodological research, nursing students` clinical performance was defined. Domains and items of the tool were determined through nursing educators`opinions (expert interviews), a sound literature review and considering assessment tools in some universities around the world. The gathered data was reviewed by the researchers and the primary tool was designed. The tool was prepared by a 5 point Likert scale. Psychometric properties of the tool were determined as follow:

\subsection{Validity:}

\subsubsection{Face validity}

To assess face validity, the tool was reviewed by five expert nurse educators. Also using item impact method, the importance of each item was calculated with the judgment of 15 experts and items with score of 1.5 and more remained in the tool.

\subsubsection{Content validity}

To assess content validity, the tool was reviewed by 15 experts. Also content validity ratio (CVR) was determined through 13 experts` judgment, and based on Lawshe`s table, items with the score of 0.54 and more remained in the tool. Content validity index (CVI) was determined by 15 experts using a four point scale described by Waltz and Bausell. The score of 0.80 was considered as the least acceptable CVI.

\subsection{Reliability:}

\subsubsection{Internal consistency}

Internal consistency was evaluated by Cronbach`s alpha. The designed tool was used by clinical educators to evaluate 30 students during their clinical education. A coefficient more than 0.75 was considered appropriate.

\subsubsection{Inter class correlation (ICC)}

ICC was calculated based on simultaneous observation made by two trained nurse educators on 15 nursing students. Correlation more than 0.80 was considered as good.

\section{Main results}

To design the clinical performance evaluation tool for nursing students` clinical performance, the definition of clinical performance was considered as activities in which a student participates in nursing care of clients to meet their health care needs. Then the primary tool with seven domains and 55 items was designed (Table1).

\subsection{Validity}

3.1.1 Face validity was determined using experts' opinions to correct the tool. The importance of each item was also calculated through item impact method based on experts` judgments. Scores of all items were more than 1.5. 
3.1.2 Content validity was determined using experts`opinions to correct the tool. CVR and CVI were also calculated based on experts` judgments. CVR score was 0.54 and more for each item, CVI for each item was at least 0.80 and for the whole tool 0.95 .

\subsection{Reliability:}

3.2.1 Cronbach`s alpha for the whole tool was 0.987 , and it was also determined for each domain (Table2).

3.2.2 Inter class correlation was 0.89 .

Table1: Domains and items of the tool

\begin{tabular}{cc}
\hline Domain & items \\
\hline Professional behavior & 11 \\
Communication & 9 \\
Nursing care plan & 9 \\
Patient education & 6 \\
Nursing report & 6 \\
Patient safety & 6 \\
Infection control & 8 \\
\hline
\end{tabular}

Table2: Cronbach`s alpha for each domain of the tool

\begin{tabular}{cc}
\hline Domain & Cronbach`s alpha \\
\hline Professional behavior & 0.935 \\
Communication & 0.937 \\
Nursing care plan & 0.955 \\
Patient education & 0.947 \\
Nursing report & 0.955 \\
Patient safety & 0.895 \\
Infection control & 0.926 \\
\hline
\end{tabular}

\section{Conclusions}

Lack of appropriate assessment tools to evaluate nursing students` performance in clinical education is a long-standing problem and ongoing researches for objective assessment tools to be used by clinical educators continues [16]. Some studies have focused on designing tools for nursing students`clinical evaluation $[1,11]$ but it seems the problem still remained a major challenge. Many studies have mentioned this problem and emphasized on the necessity of appropriate tools. Using well-defined assessment forms and scales to make the assessment structured and standardized and designing evaluation tools that are able to capture the unique and context dependent nature of clinical practice are issues under discussion $[1,17]$.

In this methodological research, a valid, reliable and objective assessment tool to evaluate nursing students` clinical performance was designed. First the main concept, clinical performance, was defined as "the totality of directed activity in which a student engages in nursing practice with consumers to meet health care needs" [18]. Based on the definition, domains and items of the tool were determined through interviews with nursing education experts and literature review. As a result, a tool with seven domains and 55 items was designed.

There have been some efforts in this area. In a study as part of a curriculum improvement for their baccalaureate nursing program, the nursing faculty, teaching in a 4-year undergraduate program identified the need for the development of a new clinical performance evaluation tool for the evaluating of undergraduate nursing students. The resultant tool more accurately appraised clinical capabilities by focusing on quality and safety in health care, and it permitted the evaluation of critical thinking skills and team communication [11].

Also the challenge of determining psychometric properties remains a major problem. Some tools are designed and used without appropriate assessment of their validity and reliability. However, key indicators of the quality of a measuring tool are the validity and reliability of the measures [19]. The designed tool in this study has good validity and reliability. In each stage of psychometry, experts viewpoints were considered to correct the tool. The determined item impact score was 1.5 and more for each item which was good [20, 21]. CVR was 0.54 and more in each item and was considered as appropriate based on 13 experts` judgments and Lawshe`s table [22]. CVI was 0.95 for the whole tool, and more than 0.80 for each item and was considered as appropriate [23]. So all items remained in the designed tool. In reliability; high internal consistency was seen with a Cronbach`s alpha of 0.987 for the whole tool and appropriate coefficients for all domains, and also inter class correlation was good with $r=0.85[24,25]$. 
In general, findings showed that the designed tool have good validity and reliability. Therefore, it could be used as an appropriate tool in clinical assessment of nursing students. It is obvious that the tool may have problems in extended use, because in this study was used in a limited population. Further investigation on the tool is necessary.

\section{References}

[1] FF Bourbonnais, S Langford, L Giannantonio. Development of a clinical evaluation tool for baccalaureate nursing students. Nurse Education in Practice 8, 1 (2008) 62-71.

[2] A Elcigi, H Yildirım Sari. Determining problems experienced by student nurses in their work with clinical educators in Turkey. Nurse Education Today 27, 5 (2007) 491-498.

[3] E Hellstrom-Hyson, G Martensson, ML Kristofferzon. To take responsibility or to be an onlooker. Nursing students' experiences of two models of supervision. Nurse Education Today. 32 , 1 (2011) 105-10.

[4] M Neary. Teaching, Assessing and Evaluation for Clinical Competence, A Practical Guide for Practitioners and Teachers. Stanley Thornes Publishers Ltd, United Kingdom, 2000.

[5] MH Oermann, KB Gaberson. Evaluation and Testing in Nursing Education. Springer, New York, 2006.

[6] PA Chesser-Smyth. The lived experiences of general student nurses on their first clinical placement: A phenomenological study. Nurse Education in Practice. 5, 6 (2005) 320-327.

[7] B McCarthy, S Murphy . Assessing undergraduate nursing students in clinical practice: Do preceptors use assessment strategies? Nurse Education Today. 28, 3 (2008) 301-13.

[8] KB Gaberson, MH Oermann. Clinical Teaching Strategies in Nursing. Springer Publishing, New York, 2007.

[9] VE Coates, M Chambers. Evaluation of tools to assess clinical competence. Nurse Education Today. 12, 2 (1992) 122-9.

[10] MR Gardner, P Dunphy Suplee. Hand Book of Clinical Teaching. Jones and Bartlett, Boston, 2007.

[11] T Walsh, N Jairath, MA Paterson, C Grandjean. Quality and safety education for nurses clinical evaluation tool. Journal of Nursing Education. 49, 9 (2010) 517-22.

[12] S Redfern, I Norman, L Calman, R Watson, T Murrells. Assessing competence to practice in nursing: a review of the literature. Research Papers in Education. 17, 1 (2002) 51-77.

[13] GS Wishinia, P Yancy, J Silva, N Kern-Manwaring. Evaluation by exception for nursing students. Journal of Nursing Education. 41, 11 (2002) 495-497.

[14] L Calman, R Watson, I Norman, S Refern, T Murrells. Assessing practice of student nurses: methods, preparation of assessors and student views. Journal of Advanced Nursing. 38, 5 (2002) 516-23.

[15] G LoBiondo-Wood, J Hober. Nursing Research, Methods and Critical Appraisal for Evidence-Based Practice. 7th Edition. Mosby- Elsevier co, St. Louis, 2010.

[16] J Donohue, SD Pelletier. An empirical analysis of a clinical assessment tool. Nurse Education Today. 11, 5 (1991) 354-362.

[17] M Hijazi. Structured continuous objective-based assessment of resident's performance at the point of care (SCOPA). Ann Saudi Med. 25, 3 (2005) 193-197.

[18] MD Bantell. Clinical evaluation methods used by baccalaureate nursing educators in traditional and nontraditional clinical areas. [Dissertation]. Columbia University, Columbia, 2005. available online: http:// www.proquest.com.

[19] CL Kimberlin, AG Winterstein. Validity and reliability of measurement instruments used in research. American Journal of Health System Pharmacist. 65, 23 (2008) 2276-2284.

[20] Y Lacasse, C Godbout, F Series. Health related quality of life in obstructive sleep apnea. European Respiratory Journal. 19,3 (2002) $499-503$.

[21] EF Juniper, GH Guyott, DL Streiner. Clinical impact versus factor analysis for quality of life questionnaire construction. Journal of Clinical Epidemiology. 50, 3 (1997) 233-238.

[22] CH Lawshe. A quantitative approach to content validity. Personnel Psychology. 28, 4 (1975) 563-575.

[23] DF Polit, Ch Tatano Beck. Essentials of Nursing Research. Appraising Evidence for Nursing Practice. 7th Edition. Lippincott-Williams \&Wilkins, Philadelphia, 2010.

[24] JL Fleiss. The Design and Analysis of Clinical Experiments, Wiley and Sons, New York, 1986. 